\title{
Bioorganic studies with rhodopsin
}

\author{
Koji Nakanishi
}

Department of Chemistry, Columbia University, New York, New York 10027 , U.S.A. and Suntory Institute for Bioorganic Research, Shimamoto-cho, Mishima-gun, Osaka 618, Japan

\begin{abstract}
An introductory review of visual transduction is followed by an account of external point charge models which were proposed to explain the red-shifted maxima in visual pigments and bacteriorhodopsin. 13-Ethylretinal, 13-propylretinal, and a retinal analog with an 8-membered ring in the side-chain yield bovine rhodopsin analogs which are bleachable but spontaneously regrow. This is a phenomenon not seen before in vitro in rhodopsins. The photoreceptor of the phototactic green algae Chlamydomonas has been found to be closely related to bovine rhodopsin but unlike bacteriorhodopsin; incubation of Chlamydomonas with retinal analogs shifts the phototaxis action spectral maxima in a manner similar to the shifts encountered in the bovine visual pigment. This is the first behavioral shift induced by analogs in a eukaryote. The studies also suggest that Chlamydomonas provides a convenient system for photoreceptor studies because of the availability of mutant strains, relative ease of experiment, and the short time needed to get results on minuscule amounts of analogs.
\end{abstract}

\section{INT RODUCT ION}

In 1933 Wald discovered that vitamin A was present in the retina (Ref. 1), and then in 1950 determined that the chromophore of the visual pigment rhodopsin is 11-cis-retinal (Ref. 2). Studies in vision have dramatically increased in recent years since these discoveries, and involve researchers in all branches of science. However, it appears that the more we study, the more complex the phenomenon of visual transduction becomes. Intimate collaboration between scientists in all disciplines is indispensable.

An introductory account of visual pigments is given (Refs. 3-6) and this is followed by unpublished studies. The visual pigments are in the photoreceptor cells. There are two types of photoreceptors, the rod/cone cells of vertebrates and the rhabdomeric cells of invertebrates. Both consist of inner and outer segments, and it is the latter which contain the photoreceptors. In the vertebrate eye, the light enters the lens (Fig. 1) and hits the neural photoreceptor cells distributed in the retina. There are two types of photoreceptors, the rod cells which are primarily in the peripheral region of the retina, and the cone cells which are clustered around the central fovea where focusing of image is sharpest. The rod outer segment (ROS) contains 1,500 stacked disks; the rhodopsin molecules, $\lambda_{\max } 500 \mathrm{~nm}$, which are responsible for black and white sensation in dim light are embedded in the disks (Fig. 1). The human retina has three types of cone cells which absorb at 450, 530 and $560 \mathrm{~nm}$ and work under bright illumination. Most animals have only one kind of visual pigment and cannot distinguish color. Rhodopsin, a hydrophobic protein comprised of 348 amino acids, traverses the disk membrane several times. Its primary structure 


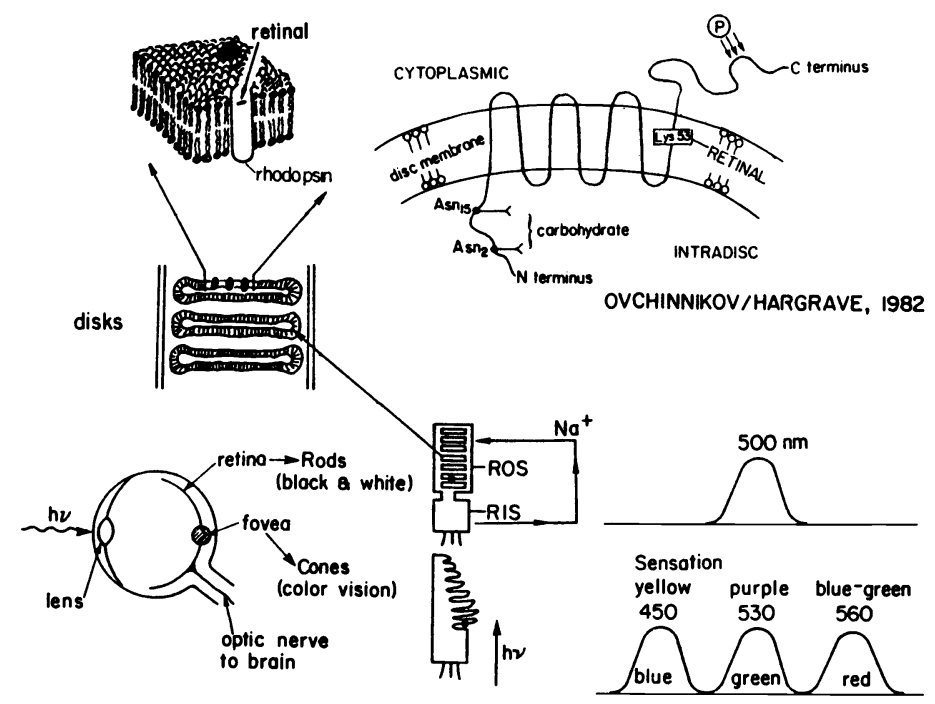

Fig. 1. The eye and photoreceptor cells. The rhodopsin molecule traverses the disk membrane (upper left and right)

was elucidated recently (Refs. 7, 8) but the tertiary structure (Ref. 9) has yet to be clarified experimentally.

Vitamin A, produced by cleavage of carotenoids, is partly converted into 11-cis-retinal; binding of this chromophore to the rod and cone opsins produces the various visual pigments (Fig. 2). Certain animals such as fish and tadpoles have the 3-dehydro chromophore in addition. Most rhodopsin studies including those described below are carried out with the bovine pigment because of its availability.

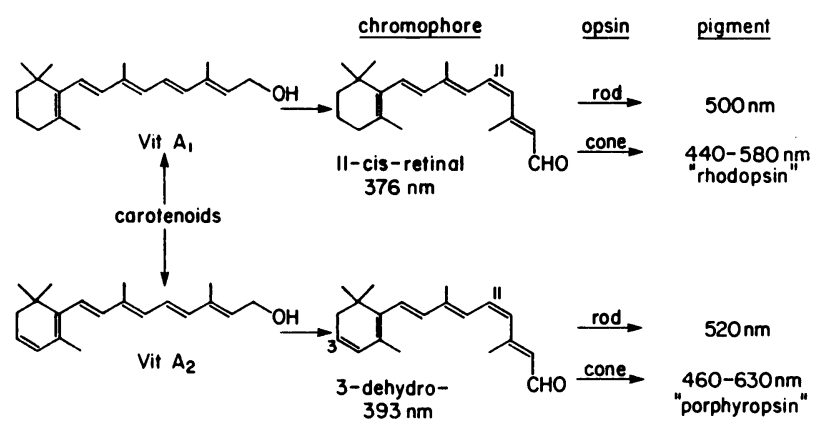

Fig. 2. Formation of visual pigments

The chromophore is bound to lysine 53 via a protonated Schiff base (Fig. 3, 1). Although the bleaching process appears to differ somewhat from animal to animal, it is generally agreed that the primary photoproduct is bathorhodopsin discovered in 1958 (Ref. 10). Subsequent steps are thermal and proceed through several intermediates terminating in trans-retinal $\underline{2}$

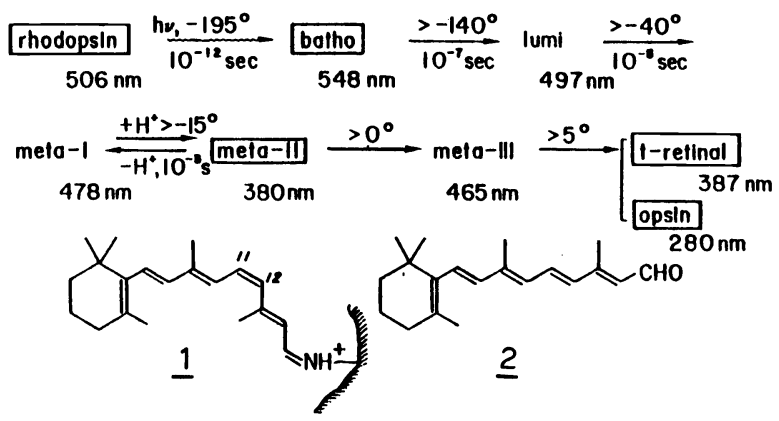

Fig. 3. Bleaching process of cattle rhodopsin $(1=$ protonated Schiff base, $\mathrm{SBH}^{+}$) 
and opsin. The light isomerizes the 11-cis-retinal moiety 1 to an unidentified retinal species contained in bathorhodopsin ( $\operatorname{Re}$. 11) within a few picoseconds (Refs. 12, 13). Bathorhodopsin, a ground state species with $35 \mathrm{kcal}$ higher energy than rhodopsin (Ref. 14), possibly stores its energy by separation of the positively charged iminium nitrogen from its counter-anion (hence the red-shift in absorption) (Ref. 15) and by internal strains within the chromophore.

The species directly involved in visual transduction is thought to be metarhodopsin-II. In the dark, sodium ions flow into the rod outer segments (ROS, Fig. 1) from the extracellular fluid and diffuse into the inner segment (RIS) where they are pumped out again by an ATP-dependent pump. Upon illumination, the sodium channel is blocked and the dark current is shut of $f$. This leads to a neural signal which is transmitted to the brain via the optic nerve, but the manner in which the change in shape of rhodopsin, which is triggered by the 11-cis to trans isomerization, relates to transduction is still unclear. Two hypotheses have been forwarded (Ref. 16). Since calcium flows out of the disk into the plasma membrane upon irradiation and since raising the calcium level shuts down the dark current, it has been proposed that calcium is the internal transmitter which stops the flow of sodium ions. In another theory, cyclic GMP is the transmitter. Namely, activated rhodopsin (meta-II) changes a protein called G-protein (or transducin) from an inactive to an active form, and this activates a phosphodiesterase which hydrolyzes cyclic GMP to GMP; the bleaching of one molecule of pigment results in the destruction of 10,000 molecules of cyclic GMP. An understanding of the visual transduction process on a molecular structural basis is one of the central problems in vision science.

\section{EXTERNAL POINT CHARGE MODELS}

The factors involved in regulating the absorption maxima of rhodopsins has been a question of major interest. Namely, the protonated Schiff base $\left(\mathrm{SBH}^{+}\right)$of retinal with $\mathrm{n}-\mathrm{BuNH}_{2}$ absorbs at $440 \mathrm{~nm}\left(22,700 \mathrm{~cm}^{-1}\right.$, in $\left.\mathrm{MeOH}\right)$ while the $\mathrm{SBH}^{+}$in visual pigments absorbs at 440-650 nm $(22,700-15,400$ $\mathrm{sm}^{-1}$ ). In bacteriorhodopsin, a light-induced proton-translocating pigment attracting great interest since its discovery in the early 70's (Ref. 17), the $\mathrm{SBH}^{+}$now absorbs at $560 \mathrm{~nm}\left(17,860 \mathrm{~cm}^{-1}\right)$. What are the factors leading to these red shifts? Based on experiments with dihydroretinals we proposed the so-called external point-charge models for the two pigments (Fig. 4, Ref. 18). First the red shifts (in $\mathrm{cm}^{-1}$ ) in going from 11-cis-retinal, $\mathrm{SBH}^{+}$with $\mathrm{n}-\mathrm{BuNH}_{2}, 440 \mathrm{~nm}$ or $22,700 \mathrm{~cm}^{-1}$, to the pigments, $\overline{\mathrm{e} . \mathrm{g}}$, bovine rhodopsin, $500 \mathrm{~nm}$ or $20,000 \mathrm{~cm}^{-1}$, were defined as "opsin shifts" (OS); in

BOVINE RHODOPSIN BACTERIORHODOPSIN JACS 7082, 7084, 7086 (1979) JACS 7945 (1980)
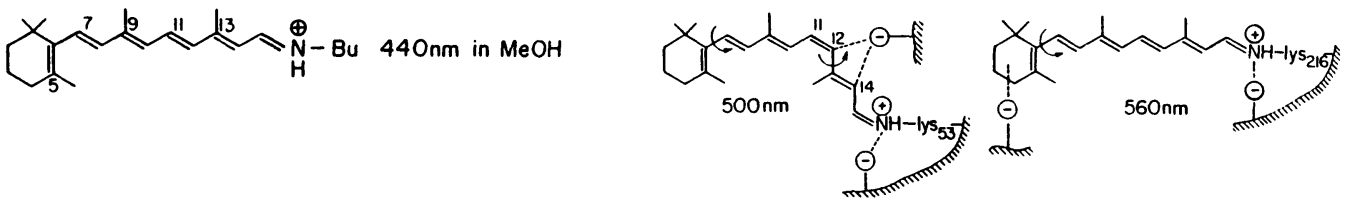

Fig. 4. External point charge models 
the example cited it is $2,700 \mathrm{~cm}^{-1}$. The $0 S$ is a value which reflects the difference between the environment of $\mathrm{SBH}^{+}$in methanol and within the binding site.

Our approach in the studies of visual pigments and the closely related bacteriorhodopsin has been to synthesize retinal analogs with modified structures, bind them to apoproteins to produce pigment analogs, and then to clarify certain aspects of natural pigments from the properties of analogs. One series of studies is the following. In the dihydroretinal series (Fig. 5) it is seen that os has the largest value of $5,300 \mathrm{~cm}^{-1}$ for

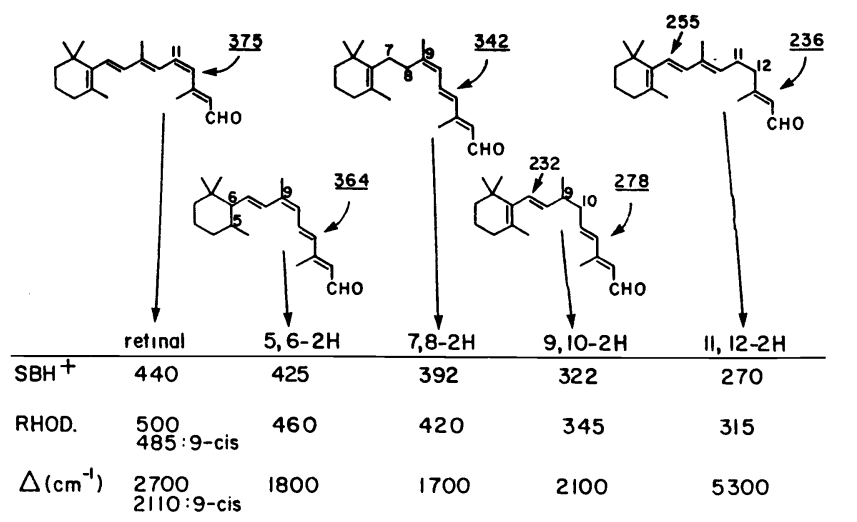

Fig. 5. Opsin shifts of bovine rhodopsins and dihydrorhodopsins

11,12-dihydroretinal, the analog with the shortest chromophore ( $\operatorname{Ref} .19$ ). Theoretical treatment of this trend (Ref. 18) coupled with further model studies (Ref. 20) led to the models shown in Fig. 4. Although the validity of these models and the identity of the external charges will not be known until the tertiary structures are clarified, they are currently the generally accepted models.

\section{SPONTANEOUS REGROWTH OF BLEACHED VISUAL PIGMENTS}

The study of visual pigments containing retinal chromophores with multiple alkyl substituents in the side-chain is likely to clarify the steric environment of the binding site; also, the extra substituents may decelerate or accelerate the formation of bathorhodopsin and other intermediates. Likewise, bacteriorhodopsin; a comparison of the proton translocation efficiency of the alkylated analogs with natural bacteriorhodopsin could possibly contribute to our understanding of this very important phenomenon. With this in mind a series of alkylated retinals has been synthesized (Ref. 22) and are being bound to form pigments. The hindered retinals were prepared according to Fig. 6 (Ref. 21); the advantage of this method is that it yields the otherwise

SYNTHESIS OF HINDERED RETINALS<smiles>CC(=O)/C=C/C1=C(C)CCCC1(C)C</smiles><smiles>CC(C)=CC(C)=CC=CC1=C(C)CCC(CO)C1(C)C</smiles>

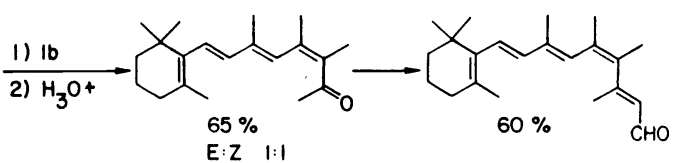

Fig. 6. Synthesis of hindered alky1 retinals 


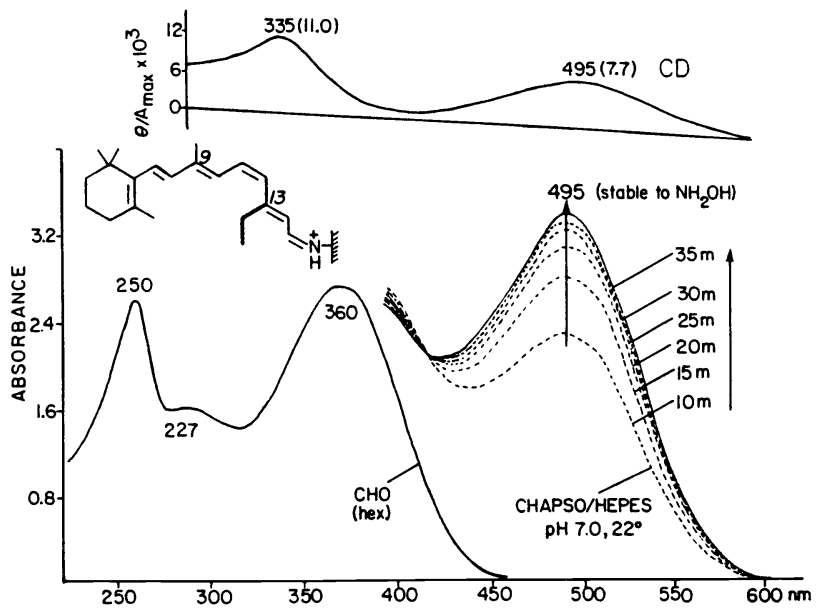

Fig. 7. UV/MS and CD spectra of 13-ethy1-11-cis-retinal and rhodopsin analog

difficult to obtain, severely hindered $Z$ isomers in fair yield. During the course of these studies a unique spontaneous regeneration was encountered. 13-Ethylretinal with maxima at 250, 277, and $360 \mathrm{~nm}$ (Fig. 7) readily forms a pigment in the detergent CHAPSO, $\mathrm{pH} 7.0$. It absorbs at $495 \mathrm{~nm}$, and exhibits two positive cotton effects in the CD spectrum. Upon irradiation with light longer than $520 \mathrm{~nm}$, it "bleaches" in one minute (Fig. 8); however, a spontaneous pigment regrowth is observed in the dark. After 48 hours it has a $485 \mathrm{~nm}$ maximum. The pigment from 13-propylretinal also behaves similarly.

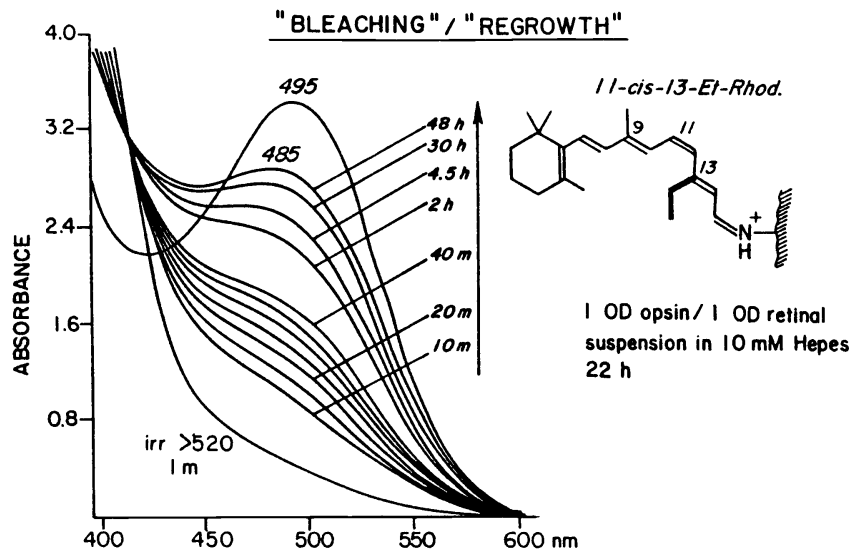

Fig. 8. "Bleaching" and regeneration of a pigment analog from 13-ethylretinal

This regrowth phenomenon can tentatively be interpreted as follows. The maximum of the regenerated species is at the long wavelength of $485 \mathrm{~nm}$ and therefore is best explained by an $\mathrm{SBH}^{+}$species; the "bleached" species on the other hand cannot be $\mathrm{SBH}^{+}$because of the lack of long wavelength absorption. Irradiation causes a double bond isomerization, but unlike natural 11-cis-retinal, the analog is not freed from the binding site because of the 13-Et or 13-Pr groups, i.e., the extra length of the alkyl group fits into a hydrophobic site thus preventing the SB linkage from being exposed and hydrolyzed. Namely, the $\mathrm{C}=\mathrm{N}$ bond simply becomes deprotonated, and hence the blue-shift, or "bleaching"; the isomerized form represents an unstable ground state so that it reisomerizes to a stabler form and becomes reprotonated to an $\mathrm{SBH}^{+}$absorbing at $485 \mathrm{~nm}$.

The 7-membered retinal analog with a fixed 11-ene (Fig. 12) as expected produced a nonbleachable pigment in vitro (Refs. 13, 23) and in vivo (rat 
and bull-frog, Ref. 24), thus showing that an 11-cis to 11-trans isomerization is a prerequisite for visual transduction. If it were possible to produce pigment analogs with 8-and 9-membered rings bridging across $\mathrm{C}-10$ and $\mathrm{C}-13$, then: (i) the larger ring may accommodate a trans-11-ene and thus provide a mimic for bathorodopsin which is generally believed to contain a highly strained transoid double bond (Ref. 25); and (ii) constraints from the ring may produce a bleachable pigment which may serve as a mimic for an intermediate further along the bleaching process (Fig. 3).

The 8- and 9-membered retinal analogs were prepared according to Fig. 9 (Ref. 26). The 8-membered analog appears to give rise to two pigments (Fig. 10, Ref. 27). Thus when incubated with bovine opsin at $18-28^{\circ}$ a species with a maximum at $430 \mathrm{~nm}$ is the major product; at $37^{\circ}$ a species
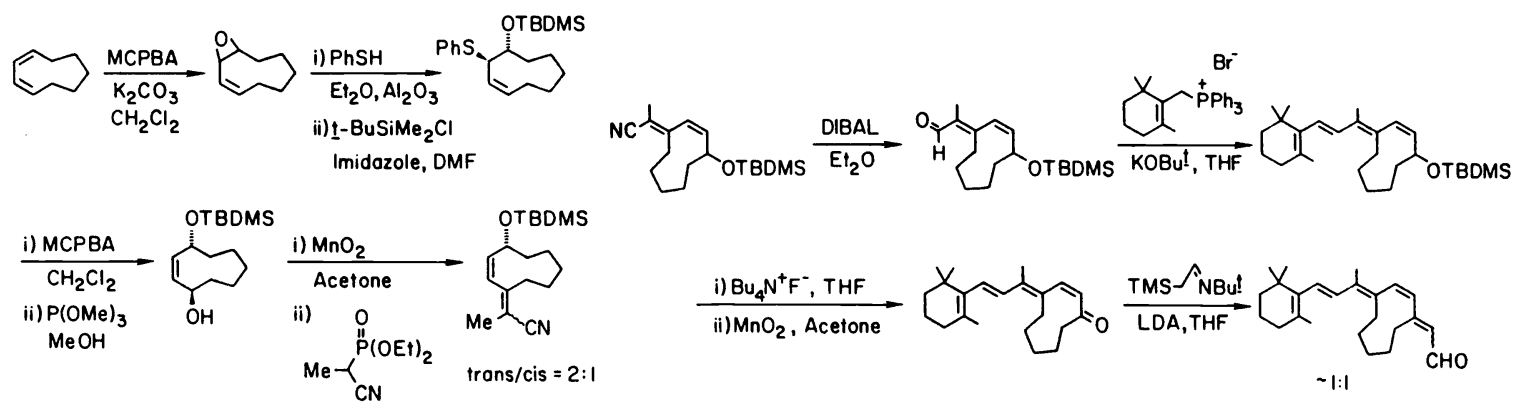

$\mathrm{NaH}, \mathrm{DME}$

Fig. 9. Synthesis of 9-membered retinal analog

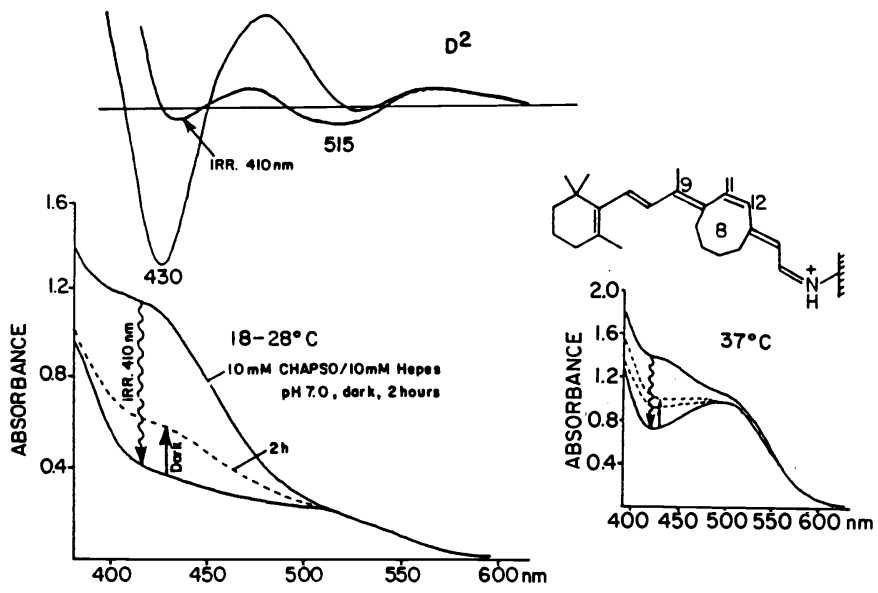

Fig. 10. Pigment formation with 8-membered retinal analog; derivative spectra are also shown

absorbing at $515 \mathrm{~nm}$ is clearly produced. Upon irradiation, bleaching of the 430 species and subsequent spontaneous regrowth occurs, but the 515 species is not bleached. The 9-membered retinal also gives a pigment, absorption maximum $407 \mathrm{~nm}$ in CHAPSO, which is now bleachable but does not regrow. Low-temperature spectroscopic studies and checking of enzymatic activations induced by bleaching and/or regrowth processes of the pigments formed from alkylated and medium-ring-containing retinals are being planned.

\section{CHLAMYDOMONAS, A NEW MODEL FOR THE STUDY OF PHOTORECEPTORS}

Chlamydomonas (Fig. 11) is a $10 \mu \mathrm{m}$ diameter aquatic organism with a single eye and two flagella which together lead to phototaxis (Ref. 28). It is widely used for studying circadian rhythm, flagella architecture and function, herbicide development, gametogenesis, photosynthesis, etc.; in 
collaboration with Prof. K.W. Foster, we have shown that this genetically well-studied eukaryote provides a convenient model for photoreception studies ( $\operatorname{Ref}$. 29).

Retinal analogs have given numerous rhodopsin and bacteriorhodopsin analogs absorbing at different wavelengths. At least 4 analogs have been shown to shift the behavioral action spectrum of Halobacterium (Ref. 30). In the following a blind mutant in which carotenoid biosynthesis is blocked at a step preceding phytoene was employed. The mutant is negatively phototactic, i.e., it swims away from light. If a retinal moiety were the chromophore, addition of retinal to the cell suspension of vegetative cells should restore phototaxis. Indeed it was found that when left overnight so that cells became gametes and incorporate the retinal, the phototaxis behavior was restored. Moreover, it was found that addition of dihydroretinals induced phototaxis with action spectra maxima closely

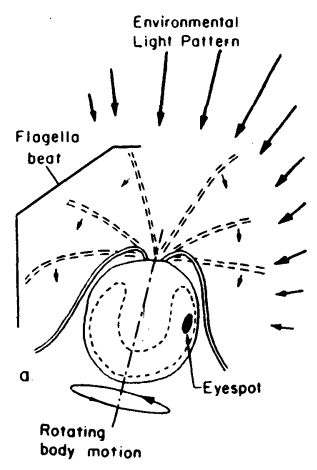

Fig. 11. Ch1amydomonas

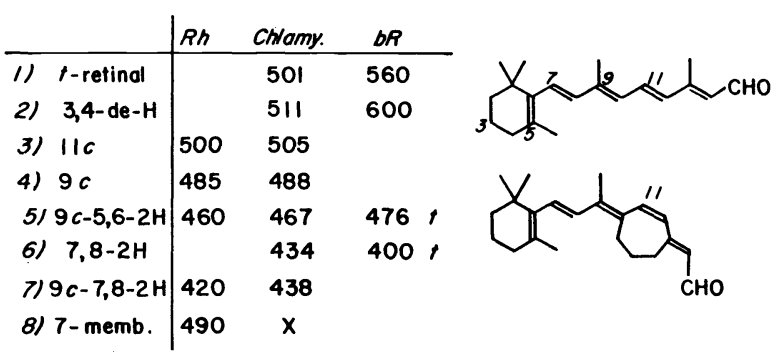

Fig. 12. Induced photoaxis in Chlamydomonas and maxima of rhodopsin, bacteriorhodopsin and analogs

resembling the absorption maxima of bovine rhodopsin but unlike those of bacteriorhodopsin (Fig. 12; see also Fig. 5). The natural action spectral maximum of Chlamydomonas is at $503 \mathrm{~nm}$, whereas the maxima after trans and 11-cis-retinal incorporation are at 501 and $505 \mathrm{~nm}$, respectively. Since these values are within experimental error it is likely that the retinals are isomerized in the dark (to 11-cis or 9-cis or trans). However, the 488 $\mathrm{nm}$ maximum caused by incorporation of the 9-cis isomer is outside the range of error and parallels the $485 \mathrm{~nm}$ of bovine pigment. These findings lead us to suspect that Chlamydomonas, an organism which separated from our evolution line about 1 billion years ago, has a pigment similar to the bovine rhodopsin ( $\operatorname{Ref} .29$ ).

Irradiation of the blind mutant leads to restoration of natural phototaxis. Furthermore, incorporated retinal analogs also restore native phototaxis when irradiated around their respective maxima, e.g., 7,8-dihydroretinal, which leads to a phototaxis action spectral maximum at around $435 \mathrm{~nm}$ (Fig. 12) induces native phototaxis most efficiently when irradiated at $435 \mathrm{~nm}$. This shows that retinals play a dual role, one is to cause phototaxis and the other is to turn on the blocked carotenoid biosynthesis ( $\operatorname{Ref} .31$ ). These are the first demonstrations of a rhodopsin photoreceptor in an alga or eukaryotic protist and also the first behavioral spectral shifts caused by exogenous synthetic retinals in a eukaryote. 
Acknowledgments - I am grateful to my colleagues who are cited in the various references. Unpublished studies were carried out by $\mathrm{Dr}$. M. Okabe, J. Termini, A.A. Croteau (hindered retinals), Drs. C.G. Caldwell, F. Derguini, C.F. Bigge (8- and 9-membered retinals), G.R. Zarrilli (Chlamydomonas) and Dr. V. Balogh-Nair and J.D. Cárriker (binding studies). The Chlamydomonas studies were carried out in Prof. K.W. Foster's laboratory, Mount Sinai School of Medicine. The studies have been supported by NIH grant EY 01253.

\section{REFERENCES}

1. G. Wald, Nature (London) 132, 316 (1933).

2. G. Wald and P.K. Brown, Proc. Natl. Acad. Sci. USA 36, 84 (1950).

3. H. Shichi, Biochemistry of Vision, Academic Press, New York, p. 280 (1983).

4. L. Packer, Ed., Methods in Enzymology, Academic Press, New York, Vol. 88 (1982).

5. V. Balogh-Nair and K. Nakanishi, "The Stereochemistry of Vision," in New Comprehensive Biochemistry, Ch. Tamm, Ed., Elsevier Biomedical

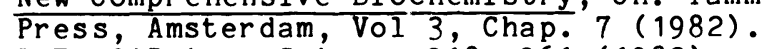

6. D.F. O'Brien, Science 218,961 (1982).

7. Y.A. Ovchinnikov, N.G. Abdulev, M.Y. Feigina, I.D. Artamonov, A.S. Zolotarev, M.B. Kostina, A.S. Bogachuk, A.I. Miroshnikov, V.I. Martinov and A. Kudelin, Bioorg. Khim. 8,1011 (1982).

8. P.A. Hargrave, J.H. McDowell, D.R. Curtis, J.K. Wang, E. Juszczak, S.-L. Fong, J.K.M. Rao and P. Argos, Biophys. Struct. Mech. 9, 235 (1983).

9. E.A. Dratz and P.A. Hargrave, Trends Biochem. Sci. 8, 128 (1983).

10. T. Yoshizawa and Y. Kito, Nature 182, 1604 (1958); T. Yoshizawa and G. Wald, ibid. 197, $1279(196 \overline{3})$.

11. R.S.H. Liu and A.E. Asato, University of Hawaii, have proposed an intriguing new model for bathorodopsin: private communication.

12. G.E. Busch, M.L. Applebury, A.A. Lamola and P.M. Rentzepis, Proc. Natl. Acad. Sci. USA 69, 2802 (1972).

13. J. Buchert, V. Stefancic, A.G. Doukas, R.R. Alfano, R.H. Callender, J. Pande, H. Akita, V. Balogh-Nair and K. Nakanishi, Biophys. J.. 43, 279 (1983).

14. A. Cooper, Nature 282, 531 (1979).

15. B. Honig, T.G. Ebre ey, R.H. Callender, U. Dinur and M. Ottolenghi, Proc. Natl. Acad. Sci. USA 76, 2503 (1979).

16. W.L. Hubbeli and M.D. Bownds, Ann. Rev. Neurosci. 2, 17 (1979).

17. D. Desterhelt and W. Stoeckenius, Nature New Biol. 233, 149 (1971).

18. B. Honig, U. Dinur, K. Nakanishi, V. Balogh-Nair, M.A. Gawinowicz, M. Arnaboldi and M.G. Motto, J. Am. Chem. Soc. 101, 7084 (1979); K. Nakanishi, V. Balogh-Nair, M. Arnaboldi, K. Tsujimoto and B. Honig, ibid. 102, 7945 (1980).

19. M. Arnaboidi, M.G. Motto, K. Tsujimoto, V. Balogh-Nair and K. Nakanishi, J. Am. Chem. Soc. 101, 7082 (1979).

20. M. Sheves, K. Nakanishi and B. Honig, J. Am. Chem. Soc. 101, 7086 (1979); M.G. Motto, M. Sheves, K. Tsujimoto, V. Balogh-Nair and K. Nakanishi, ibid: 102, 7947 (1980); M. Sheves and K. Nakanishi, ibid. $105,4033(\overline{1983})$.

21. A.A. Croteau and J. Termini, Tetrahedron Lett. 2481 (1983).

22. M. Okabe and J. Termini, unpublished.

23. H. Akita, S.P. Tanis, M. Adams, V. Balogh-Nair and K. Nakanishi, J. Am. Chem. Soc. 102, 6370'(1980); B. Mao, M. Tsuda, T.G. Ebrey, H. Akita, V. Balogh-Nair and K. Nakanishi, Biophys. J. 35, 543 (1981).

24. R. Crouch, B.R. Nodes, J.I. Perlman, D.R. Pepperberg, H. Akita and K. Nakanishi, Invest. Ophthalm. Vis. Sci. 25, 419 (1984).

25. G. Eyring, B. Curry, R. Mathies, R. Fransen, I. Palings and J. Lugt enburg, Biochemistry 19, 2410 (1980); G. Eyring, B. Curry, A. Broek, J. Lugtenburg and $\bar{R}$. Mathies, ibid. 21,384 (1982).

26. C.G. Caldwell, F. Derguini, C.F. Bigge, unpublished.

27. V. Balogh-Nair, unpublished.

28. K.W. Foster and R.D. Smyth, Microbiol. Rev. 44, 572 (1980).

29. K.W. Foster, J. Saranak, N. Patel, G.R. Zarrilí, M. Okabe, T. Kline and K. Nakanishi, Nature, in press.

30. A. Schimz, W. Sper ling, E. Hildebrand and D. Kohler-Hahn, Photochem. Photobiol. 36, 193 (1982).

31. K.W. Foster, G.R. Zarrilli, et al., unpublished. 